\title{
Curcumin as a Complementary Treatment in COVID-19
}

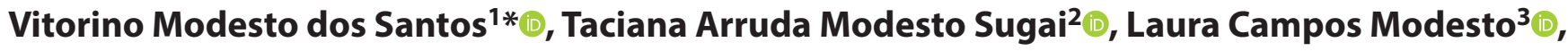 \\ Julia Campos Modesto ${ }^{4}$ \\ 'Department of Medicine, Armed Forces Hospital, Brasília-DF, Brazil; \\ ${ }^{2}$ American Society of Neurophysiology, and Dermatologist of Brasília-DF, Brasília-DF, Brazil; \\ ${ }^{3}$ University Center of Brasília-DF, Brasília-DF, Brazil; \\ ${ }^{4}$ Catholic University of Brasília, Brasília-DF, Brazil \\ Email: vitorinomodesto@gmail.com
}

Dear Editor, Curcumin is an herbal spice and coloring food additive extracted from Curcuma longa that has been evaluated as a complementary tool in the scenery of COVID-191-8. C. longa (turmeric) is a plant used as a traditional Indian medicine with antiviral, analgesic, antimicrobial, antiproliferative, and anti-inflammatory properties ${ }^{1-8}$. Moreover, turmeric is one of the most popular spices worldwide, mainly in India ${ }^{1,25,7,8}$. Curcumin chemoprotection and cytoprotection are due to the free radicals scavenging, and also to the antioxidant, antiinflammatory, anticancer, and antimicrobial actions ${ }^{1-8}$. Moreover,itcanexertantithromboticeffectsviatheinhibition of thrombin and FXa, and might be a complementary option to prevent post-Covid thromboembolism ${ }^{2,3,5,8}$. Curcumin can modify pro-inflammatory cytokines, apoptotic proteins, cyclooxygenase, endothelin-1, malondialdehyde, phosphorylase kinase, glutathione, prostaglandin, C-reactive protein, pepsinogen, transferrin receptor, and the transforming growth factor ${ }^{1}$. Curcumin can regulate molecules related to the inflammatory mechanisms, including cellular pathways, transcription factors, chemokines and cytokines, kinases, enzymes, and regulators of inflammasomes ${ }^{2,3,6,8}$. Curcumin has usefulness to countervail toxic manifestations of medicines as anti-tuberculosis, chemotherapy, analgesics, psychiatric and anesthetic drugs; as well as substances heavy metals, insecticides, nicotine, benzopyrene, alcohol, and aflatoxins ${ }^{1}$. Researchers have been actively seeking for some really effective prophylaxis and treatment to employ in SARS-CoV-2 infection ${ }^{2,8}$. Until consistent data of the studies on a definite treatment schedule for COVID-19, the evidence suggests curcumin as a promising alternative prophylactic management ${ }^{2,8}$. Curcumin may have antiviral activity against SARS-CoV-2 by the envelope disruption; action in viral membrane proteins; inhibition of proteases; antiviral responses induction; and targeting the NF- $\kappa \mathrm{B}$, inflammasome, IL- 6 trans-signaling, and HMGB1 pathways ${ }^{2}$. The novel genome research utilizing Clustered Regularly Interspaced Short Palindromic Repeats Cas9 (CRISPR-Cas9) revealed that the High Mobility Group Box 1 (HMGB1) is a significant pro-viral host factor in SARS-CoV-2 infection ${ }^{8}$. This non-histone nuclear protein can bind with DNA and regulate transcription; its major role in danger-related molecular patterns is to enhance the inflammatory responses by binding to Tolllike receptors and the activation of the inflammasome ${ }^{2,8}$. The severity of COVID-19 is directly related to cytokine release syndrome (CRS), and authors have described the role of inflammasome activation involved in driving CRS ${ }^{2}$. Many studies confirmed the potency of curcumin to block inflammasome activation; this property is due to curcumin (diferuloylmethane), the most abundant bioactive pharmacological of curcuminoids; bisdemethoxycurcumin, or demethoxycurcumin ${ }^{2,6,8}$. HMGB1 antagonists might be potential alternatives for protection against SARS-CoV-2

${ }^{*}$ Author for correspondence 
infection, reducing HMGB1 expression and the mediated proinflammatory responses ${ }^{2}$. Curcumin may inhibit SARS-CoV 3CL protease activity, vital for viral replication; and interacts with S protein and ACE2 protein intervening on the viral entry in lung cells ${ }^{2,8}$. Then, it may protect from COVID-19 by down-regulating the ACE2 expression ${ }^{2,4,5,7,8}$. As a promising agonist of transcription factor NRF2, curcumin can activate the NRF2 pathway in the lung of mice, an indication of antiviral activity against SARSCOV $-2^{2}$. The increased survival of infected mice treated with curcumin was related to lower levels of interleukins (IL-1beta and IL-6) and tumor necrosis factor alpha in bronchopulmonary and blood samples ${ }^{2}$. Curcumin is an adjuvant to control risk factors for COVID-19 as immunosuppression; diabetes; hypertension; heart, lung, cerebrovascular, and renal diseases; and cancer ${ }^{1,3-6}$. Worth of note, curcumin may be also effective against human influenza A virus, Respiratory Syncytial virus infection, SARS-CoV in vitro, as well as other acute and chronic respiratory disorders and in sepsis, by mechanisms involving the oxidative stress ${ }^{2}$.

Pawar et al. studied the effects of associated curcumin/piperine in the management of COVID-19; and the evolution of 70 controls under conventional COVID-19 treatment and probiotics was compared to that of 70 patients receiving the conventional treatment plus association twice daily ${ }^{3}$. The authors concluded that curcumin/piperine can improve the COVID-19 management, and their use reduced the time of hospitalization and the number of deaths ${ }^{3}$. SaberMoghaddam et al. performed a nonrandomized trial about the use of curcumin in hospitalized COVID-19 patients ${ }^{4}$. During 2 weeks 41 patients were evaluated: 21 used nano-curcumin (curcuminoids $40 \mathrm{mg}$ as nanomicelles, two capsules twice daily), and 20 patients of the control group $^{4}$. No patients died in both groups as well as none of the treatment group had clinical deterioration, which occurred in $40 \%$ of those of the control group during follow-up ${ }^{4}$. The authors commented that over 300 clinical trials showed protective effects of curcumin on cardiovascular, pulmonary, metabolic, and liver diseases, besides cancer ${ }^{4}$. They concluded that nano-curcumin can be useful to COVID-19 control without significant adverse reactions, and contribute to the reduction of hospital permanence of mild to moderate cases ${ }^{4}$.

Based on the data here commented, authors suggest more clinical trials to evaluate the curcumin preventive and therapeutic role as an alternative or complementary medication against COVID-19.

\section{References}

1. Elshama SS. The therapeutic and protective role of curcumin in drugs and chemicals intoxication. J Health Sci Res 2019;4:17-24.

2. Hassaniazad M, Eftekhar E, Inchehsablagh BR, Kamali H, Tousi A, Jaafari MR, et al. A triple-blind, placebocontrolled, randomized clinical trial to evaluate the effect of curcumin-containing nanomicelles on cellular immune responses subtypes and clinical outcome in COVID-19 patients. Phytother Res 2021;35:6417-27.

3. Pawar KS, Mastud RN, Pawar SK, Pawar SS, Bhoite RR, Bhoite RR, et al. Oral curcumin with piperine as adjuvant therapy for the treatment of COVID-19: A randomized clinical trial. Front Pharmacol 2021;12:669362.

4. Rattis BA, Ramos SG, Celes MR. Curcumin as a potential treatment for COVID-19. Front Pharmacol 2021;12:675287.

5. Saber-Moghaddam N, Salari S, Hejazi S, Amini M, Taherzadeh Z, Eslamet S, et al. Oral nano-curcumin formulation efficacy in management of mild to moderate hospitalized Coronavirus disease-19 patients: An open label nonrandomized clinical trial. Phytother Res 2021;35:2616-23.

6. Saeedi-Boroujeni A, Mahmoudian-Sani MR, Bahadoram M, Alghasi A. COVID-19: A case for inhibiting NLRP3 inflammasome, suppression of inflammation with curcumin? Basic Clin Pharmacol Toxicol 2021;128:37-45.

7. Suravajhala R, Parashar A, Choudhir G, Kumar AA, Malik B, Nagaraj VA, et al. Molecular docking and dynamics studies of curcumin with COVID19 proteins. Netw Model Anal Health Inform Bioinform 2021;10:44.

8. Thimmulappa RK, Mudnakudu-Nagaraju KK, Shivamallu C, Subramaniam KJ, Radhakrishnan A, Bhojraj S, et al. Antiviral and immunomodulatory activity of curcumin: A case for prophylactic therapy for COVID-19. Helion 2021;7:e06350. 